\title{
HISTORICIDAD E IRREVERSIBILIDAD EN LA CONCEPCIÓN PRIGOGINIANA Y AGUSTINIANA DEL TIEMPO
}

\section{EDUARDO IBÁNEEZ*}

\section{Un problema epistemológico pendiente}

¿Es pensable la unidad del tiempo? ¿Es posible articular de algún modo el tiempo existencial de la filosofía y el tiempo objetivo de la física? Se trata, sin duda, de un problema epistemológico no resuelto, y apenas planteado por la filosofía. En general, la postura moderna y aun contemporánea ha consistido en explicitar el hiato y la dicotomía entre las dos ideas de temporalidad, como si se tratara de nociones distintas e irreconciliables. Autores como Hegel, Heidegger, Gadamer, Bergson, Paul Ricoeur y muchos otros, han comprendido el tiempo histórico ontológicamente, aunque también han existido posturas mecanicistas en la filosofía, como por ejemplo la de Descartes. Mi planteo consiste en afirmar que es factible indagar la articulación de "los dos tiempos", que, aunque abordados desde dos perspectivas distintas, concuerdan en las nociones de "irreversibilidad e historicidad". En particular, la idea agustiniana de "extensio animi", aunque trasunta una concepción filosófica espiritualista e interiorista, no es contradictoria con la noción prigoginiana de "flecha del tiempo", que refleja sin embargo una concepción científica naturalista e inmanentista. Y no lo es porque ambas coinciden en la dirección del tiempo (o irreversibilidad), en la orientación bacia el futuro de la temporalidad. Esta coincidencia posibilita la articulación. No se trata solamente de señalar que ambos autores coinciden en la atención

\footnotetext{
* Profesor de la Facultad de Ciencias de la Educación de la Universidad Católica de SantaFe (invest13@gigared.com).

1 Quiero expresar mi gratitud al Dr. Anibal Fornari, cuyas originales sugerencias, orientaciones bibliográficas, críticas y correcciones efectuadas a este trabajo, iluminaron la articulación mencionada. En particular, le debo la idea de vincular las nociones de "historicidad" e "irreversibilidad" con la de "aconteciniento".
} 
prestada al tema del tiempo, sino de destacar que ambos concuerdan en la direccionalidad del tiempo. Aunque esto, obviamente, no anula las diferencias de sus respectivas posturas o concepciones.

Ya se trate del tiempo físico, objetivamente medible, o de la percepción bumana del tiempo, es decir, del tiempo "externo" o "interno", ambos remiten a las ideas de historicidad e irreversibilidad y están vinculados con la flecha del tiempo, como sostiene Prigogine. Históricamente aquella articulación comienza a percibirse recién a partir de los avances y descubrimientos de la termodinámica de los procesos irreversibles alejados del equilibrio y de la teoría del caos, es decir, de las denominadas "ciencias del devenir" del siglo XX. Resulta enteramente comprensible que la filosofía moderna, anterior a estos descubrimientos, alegara que se trata de dos tiempos distintos e inconmensurables, ya que la dinámica clásica se refería fundamentalmente a un tiempo geométrico y reversible mientras que la filosofía hacía referencia al tiempo irreversible $e$ bistórico en que se desenvuelven los acontecimientos humanos. La situación cambia fundamentalmente a partir de las ciencias del devenir: ciencia y filosofía ya no están condenadas a sostener dos concepciones antagónicas de la temporalidad: tiempo físico reversible versus tiempo humano irreversible. Este hecho favorece, a mi entender, la posibilidad de una articulación que actualmente apenas se vislumbra.

Aunque San Agustín alude esencialmente al "tiempo del alma humana" y Prigogine al "tiempo de los procesos físicos", ambos conceptos se relacionan estrechamente con la flecha del tiempo. Intentaré mostrar que la redefinición del tiempo elaborada por Prigogine para distanciarse de la idea newtoniana, permite un acercamiento a la noción agustiniana del tiempo al menos en un sentido muy claro: el de la irreversibilidad y la bistoricidad. $\mathrm{O}$ dicho de otro modo: la introducción de la flecha del tiempo en la física asemeja el mundo natural y el mundo humano eng tanto y en cuanto ambos son bistóricamente irreversibles. Probablemente esta semejanza constituya una condición necesaria, aunque no suficiente, para la posible articulación del tiempo físico y humano. Es decir, una condición sine qua non para abordar el problema epistemológico pendiente.

Durante aproximadamente tres siglos, la física mantuvo contra viento y marea la reversibilidad de las ecuaciones dinámicas que describían el movimiento. En particular, la noción newtoniana de "trayectoria" concibe masas puntuales sometidas a fuerzas externas que producen movimientos 
reversibles. Las ecuaciones diferenciales que describen dichos movimientos en el seno de sistemas dinámicos estables y homogéneos, relativamente aislados, son invariantes respecto a la inversión del pasado y el futuro, es decir, son reversibles en el tiempo. Se instauró así un abismo infranqueable entre el dominio de la naturaleza, descrito por una ciencia que excluía la irreversibilidad, y el ámbito de lo humano, dominio abordado por una filosofía que hacía de la temporalidad la dimensión esencial de la existencia humana, y de la historicidad, una propiedad fundamental inherente al acaecer social.

Para Prigogine, el tiempo surge del no-equilibrio: la irreversibilidad es una consecuencia de la inestabilidad que caracteriza a la inmensa mayoría de los procesos de la naturaleza. Únicamente considerando la complejidad de los sistemas dinámicos y termodinámicos es posible evidenciar la flecha del tiempo.

La redefinición del tiempo efectuada por Prigogine discrepa con la noción de reversibilidad de Newton, y concuerda con la idea agustiniana de irreversibilidad, al menos en el sentido restringido de la orientación o direccionalidad del tiempo. Esto no implica la eliminación de las diferencias de enfoque entre ambos autores, porque mientras el teólogo aborda el tiempo desde la doble perspectiva de la distentio animi y la intentio animi, el científico lo visualiza desde la perspectiva del alejamiento del equilibrio y la complejidad. Para ambos, sin embargo, historicidad e irreversibilidad son nociones inherentes al concepto de temporalidad.

\section{La concepción agustiniana del tiempo}

En el Libro XI de las Confesiones, San Agustín efectúa una serie de profundas y minuciosas meditaciones acerca del tiempo. La percepción del mismo como continua fuga, y a la par, como dimensión constitutiva del ser humano, lo conduce a realizar un conjunto de reflexiones en las que se esfuerza por desentrañar la estructura profunda y el sentido último de la temporalidad, tal como es vivenciada originariamente.

Esta aguda conciencia del tiempo en tanto experiencia vital preteórica o prelógica le dificulta "definir" explícitamente el tiempo, fijarlo en conceptos "claros y distintos", cristalizarlo en un lenguaje categorial estático estableciendo límites tajantes y demarcaciones precisas, "detenerlo" en una definición. Por eso, Paul Ricoeur dirá que Agustín lleva a lenguaje su 
meditación sobre el tiempo mediante la forma gramatical narrativa, toda vez que la experiencia de la temporalidad posee un carácter narrativo. Esta forma es la más adecuada para expresar la diacronía: no un lenguaje sin tiempo que define y fija, sino un lenguaje temporal que cuenta y desenvuelve, que desarrolla una historia. Las Confesiones serían, en la interpretación de Ricoeur, una narración cuyo destinatario es Dios: "Resulta justificado el hecho de leer a Agustín desde esta perspectiva si consideramos que las propias Confesiones se presentan como una especie de 'narración' dirigida a Dios"1.

Después de situar el comienzo del tiempo en el acto de la creación divina, San Agustín admite azorado que el tiempo es una cuestión paradójica, toda vez que es un concepto oscuro a la razón explicativa, pero muy claro a la vivencia interior, porque se patentiza en la experiencia vital. El tiempo es a la vez algo familiar y extraño, cercano y lejano. Este doble carácter de opacidad lógica y transparencia experiencial (experimento en mi vida el paso del tiempo como lo más familiar y originario, lo más directo e inequívoco) desemboca en la célebre frase agustiniana que afirma saber qué es el tiempo pero no poder explicarlo: lo sabe porque lo vivencia, pero no puede explicitarlo, es decir, no puede volcarlo en un lenguaje claro y comunicativo:

“¿Qué es, pues, el tiempo? ¿Quién podrá explicar esto fácil y brevemente? ¿Quién podrá comprenderlo con el pensamiento, para hablar luego de él? Y, sin embargo, ¿qué cosa más familiar y conocida mentamos en nuestras conversaciones que el tiempo? Y cuando hablamos de él, sabemos sin duda qué es, como sabemos o entendemos lo que es cuando lo oímos pronunciar a otro. ¿Qué es, pues, el tiempo? Si nadie me lo pregunta, lo sé; pero si quiero explicárselo al que me lo pregunta, no lo sé" 2 .

Paul Ricoeur destaca que esta paradoja agustiniana va unida a otras dos. La que señala que el tiempo no es, pues el futuro no es todavía, el pasado ya no es, y el presente fluye continuamente, corre como un río o un puro movimiento inacabado. Y la que señala la dificultad de medir el tiempo, ya que no es posible medir lo que todavía no es (el futuro) ni lo que ya no es (el

\footnotetext{
${ }^{1}$ Ricoeur Paul, Historia y narratividad, Paidós, Barcelona, 1999, p. 145.

${ }^{2}$ San Agustín, Confesiones, Lumen, Buenos Aires, 1996, Libro XI, p. 203. He escogido este texto y no la conocida edición bilingüe de la B.A.C. porque considero que la traducción es muy adecuada y porque incluye notas, apostillas y comentarios de sumo interés.
} 
pasado), sino solamente lo que es, lo que es abora (el presente). Pero el presente carece de extensión, no tiene un "espacio temporal", un intervalo en el cual ser lo que es; el presente en cuanto tal es lo absolutamente efímero, lo instantáneo, y siendo esto así, ¿cómo medirlo?

Así pues, según Ricoeur, tres son las paradojas enunciadas por San Agustín: el tiempo es lo más familiar y cercano, y a la vez, lo más extraño; el tiempo es y no es; lo único que se podría medir, el presente, no se puede medir porque carece de extensión.

"En primer lugar, cuando confiesa, al comienzo de su exposición, que el tiempo es, para nosotros, la experiencia más familiar y, al mismo tiempo, la más opaca. Es de sobra conocida la famosa paradoja citada por tantos y tantos escritores después de Agustín, entre los que se encuentran principalmente Husserl y Heidegger: ‘ $¿ Q u e ́$ es, por tanto, el tiempo? Si nadie me lo pregunta, lo sé; pero cuando trato de explicarlo, ya no lo sé' (XI,14). Esta paradoja, a su vez, va unida a otras dos: el tiempo no es, pues el futuro no es todavía, el pasado ha dejado de ser y el presente pasa continuamente. Sin embargo, decimos algo positivo sobre el tiempo cuando señalamos que el futuro será, que el pasado ha sido o que el presente es ahora. Pero la paradoja resulta aún más compleja cuando tratamos de medir d tiempo o cuando hablamos de un período corto o largo del mismo y, sin embargo, nos damos cuenta de que "el presente carece de extensión"' 3 .

Estas paradojas, por su oscuridad analítica, nos impiden abordar el problema del tiempo en tanto dimensión constitutiva de la experiencia humana, y hacen patente el hecho de que la bistoricidad de la experiencia, su irreversibilidad esencial, sólo puede volcarse en lenguaje mediante la función narrativa, es decir, únicamente puede comunicarse mediante el acto de contar una bistoria:

"Estas paradojas, por así decir, nos impiden acceder directamente al tiempo como dimensión de la experiencia humana. Esa opacidad lógica pone de manifiesto el hecho de que la historicidad de la experiencia humana sólo puede llevarse al lenguaje mediante la narratividad..." 4 .

Examinaré ahora, más detalladamente, la segunda y tercera paradoja.

\footnotetext{
${ }^{3}$ Ricoeur Paul, ed cit, p. 145.

4 Idem, p. 146.
} 
Pasado y futuro no son, y en cuanto al presente, que carece de extensión, su ser consiste en dejar de ser, en pasar, o fluir. En caso contrario, no sería ya presente sino eternidad, que es la dimensión intemporal propia de Dios:

"Pero aquellos dos tiempos, pretérito y futuro, ¿cómo pueden ser, si el pretérito ya no es y el futuro todavía no es? Y en cuanto al presente, si fuese siempre presente y no pasase a ser pretérito, ya no sería tiempo, sino eternidad. Si, pues, el presente, para ser tiempo es necesario que pase a ser pretérito, ¿cómo decimos que existe éste, cuya causa o razón de ser está en dejar de ser, de modo tal que no podemos decir con verdad que existe el tiempo sino en cuanto tiende a no ser?" 5.

Es decir, el ser del tiempo consiste en dejar de ser porque, no siendo el futuro ni el pasado, el presente tampoco es, ya que se compone de instantes, de partículas fugitivas que carecen de extensión, que "huyen" del futuro hacia el pasado. Si se quiere, el presente es concebido como una especie de cedazo que filtra ese fluir pero que no lo detiene, no lo "frja": ni una sola gota de tiempo queda atrapada en el cedazo. El futuro pasa a ser pasado a través del presente, cuya instantaneidad es indivisible por carecer de extensión.

" $\mathrm{Si}$, pues, hay algo de tiempo que se pueda concebir como indivisible en partes, por. pequeñisimas que éstas sean, sólo ese momento es el que debe decirse presente; el cual, sin embargo, vuela tan rápidamente del futuro al pasado, que no se detiene ni un instante siquiera. Porque, si se detuviese, podría dividirse en pretérito y futuro, y el presente no tiene espacio alguno"6.

La tercera paradoja se refiere al problema de la medición del tiempo. Para Agustín, ni pasado ni futuro, en cuanto tales, son medibles, ya que, en rigor, no son, y no siendo, mal podrían ser mensurables. Lo único que puede medirse es el presente, pero este carece de extensión, no tiene espacio o intervalo alguno, y así las cosas, ¿qué medir del presente? No el presente en sí mismo, nos dirá Agustín, sino el presente del pasado, la buella que las cosas dejaron en la memoria, el trazo de una impresión o afección -affectio- dejado por las cosas que pasan y no retornan, pero que rescatamos -es decir, rememoramos- en el presente. Se mide en tanto y en cuanto se siente, se experimenta en el alma el paso del tiempo:

${ }^{5}$ San Agustín, ed.cit., p. 203.

${ }^{6}$ Idem, Cap. XV, Libro XI, p. 204. 
"Ciertamente nosotros medimos los tiempos que pasan cuando sintiéndolos los medimos (...) Porque cuando pasa el tiempo puede sentirse y medirse; pero cuando ha pasado ya, no puede, porque no existe" .

Lo que se mide es, pues, lo que se recuerda: no las cosas mismas, que ya han pasado, sino las imágenes que las cosas dejaron en el alma, la huella de su tránsito. Lo que ya no existe, existe aún en la memoria, en el presente del pasado, y es esta duración la que se mide, toda vez que solamente se puede medir lo que de algún modo todavía es, no lo que ya no es (el pasado) ni lo que aún no es (el futuro). En términos del propio Agustín:

"Cierto que, cuando se refieren a cosas pasadas verdaderas, no son las cosas mismas que han pasado las que se sacan de la memoria, sino las palabras engendradas por sus imágenes, que pasando por los sentidos imprimieron en el alma como su huella"8.

En síntesis, se mide la affectio, lo cual es posible merced a la duración, a la permanencia del pasado en el presente (memoria).

Ahora bien, para San Agustín, el tiempo tiene una existencia más subjetiva que objetiva, en el sentido de que la temporalidad existe más propiamente en el alma, en cuanto es sentida o experimentada vivencialmente como tal, y sólo allí, en el alma, puede medirse. Lo que existe en realidad es el presente del pasado (el recuerdo), el presente del futuro (la expectación, la espera) y el presente del presente (la atención), y estas tres cosas existen en el alma. Fuera de ella, el tiempo no es nada:

"Pero lo que ahora es claro y manifiesto es que no existen los pretéritos ni los futuros, ni se puede decir con propiedad que son tres los tiempos: pretérito, presente y futuro; sino que tal vez sería más propio decir que los tiempos son tres: presente de las cosas pasadas, presente de las cosas presentes y presente de las cosas futuras. Porque éstas son tres cosas que existen de algún modo en el alma, y fuera de ella yo no veo que existan: presente de cosas pasadas (la memoria), presente de cosas presentes (visión) y presente de cosas futuras (expectación)" ".

Paul Ricoeur verá en esta triple estructura del presente vivido

\footnotetext{
7 Idem, p. 205.

8 Idem, p. 206.

9 Idem, p. 207.
} 
(recuerdo, atención y expectativa) la condición de posibilidad de la estructura de la narración, o trama, y por lo tanto, también de la historia, que es siempre historia contada, narrada. Sin embargo, admite que San Agustín no ha resuelto aún la paradoja de la extensión del tiempo, del periodo o intervalo, ya que la triple estructura del presente y su dialéctica recaen finalmente en el propio presente, que carece de extensión. En la última parte de su meditación, Agustín utiliza el concepto de distensio animi para referirse al tiempo en tanto "prolongación o distensión del alma". El tiempo sería algo así como una "extensión" del alma, un "entretenerse y durar" del espíritu. Dice Ricoeur: "En este punto, Agustín introduce la audaz noción de distensio animi, es decir, la idea de la 'prolongación del alma"'10.

Esta idea implica que el tiempo será medido en el alma, y sólo allí:

"En ti, alma mía, mido los tiempos (...) En ti -repito- mido los tiempos. La afección -affectionem- que en ti producen las cosas que pasan -y que, aun cuando hayan pasado, permanece- es la que yo mido del presente, no las cosas que pasaron para producirla: ésta es la que mido cuando mido los tiempos. Luego o ésta es el tiempo, o yo no mido el tiempo"11.

La claridad conceptual de Agustín, en este punto, es meridiana: lo que mido en el alma es la afección o impresión que las cosas dejaron en ella al pasar. Es la duración del recuerdo en la memoria lo que mido realmente: esa distensión o prolongación del alma.

La propia vida está concebida como una distensión, y Agustín recomienda vivir "olvidado de las cosas pasadas, y no distraído en las cosas futuras y transitorias, sino extendido en las que están delante de nosotros"12, puesto que de este modo, siguiendo los Salmos, el hombre se deleita en Dios, puede contemplar la eternidad divina que "no viene ni pasa", es eternamente. Pero aquí es necesario advertir que San Agustín introdujo otra "dirección" de la temporalidad, otra orientación: junto a la distentio animi (o presente del presente: la atención) y a la extensio animi (o presente del futuro: la expectación), aparece la intentio animi, la dirección del tiempo hacia el yo profundo, hacia la interioridad de la subjetividad donde el hombre encuentra

\footnotetext{
10 Ricoeur Paul, ed.cit., p. 146.

11 San Agustín, ed.cit., p. 212.

12 Idem, p. 214.
} 
"lo eterno" en él. Si con la extensio animi el hombre se eleva al futuro, con la intentio animi se sumerge en la propia eternidad, en la dimensión divina de su interioridad.

El tiempo tendría, de este modo, dos "ejes": uno horizontal y otro vertical. En el eje horizontal de la temporalidad existen las tres dimensiones ya señaladas: presente del pasado, presente del presente y presente del futuro (o, indistintamente, memoria, atención y expectativa). En el eje vertical, existe solamente la dimensión de eternidad en el hombre: la intentio animi. Jean Guitton ${ }^{13}$ expresa claramente que las dos dimensiones más importantes de estas cuatro son la extensio animi y la intentio animi, es decir, la "extensión del presente hacia el futuro"14 y "la intención hacia la eternidad", ese doble movimiento por el cual, en cada instante, el hombre se extiende hacia el porvenir y se intiende hacia lo eterno de sí mismo en sí mismo:

"San Agustín veía en el tiempo dos direcciones: de una parte, la extensio, la extensión que hace que yo tienda hacia lo que todaría no es: es la dirección, el sentido del deseo. Pero existe otra dirección: la intentio, gracias a la cual no me elevo hacia e futuro, sino hacia la eternidad de mí mismo (...) En cada momento, me extiendo hacia el futuro y me intiendo hacia la eternidad" 15.

Guitton había aclarado antes que la extensio y la intentio son las dos dimensiones más importantes de las cuatro existentes, porque el pasado, como tal, ya ha sido, y el presente en cuanto tal es un puro fluir evanescente que se escurre como el agua entre los dedos, con lo cual sólo queda en pie el presente del futuro (extensio) y la intemporalidad (intentio):

"Entre estas cuatro dimensiones, tres horizontales y una vertical, que componen la estructura del tiempo, las dos más importantes en épocas de cambio y de mutación en las que el tiempo pasa un umbral, son el futuro y el intemporal; el pasado cumplido y el presente evanescente quedan en segundo plano" 16 .

${ }_{13}^{13}$ Guitton Jean, Historia y destino, Rialp, Madrid, 1977, p. 214.

${ }^{14}$ Extensión que vincularé a la noción prigoginiana de irreversibilidad, toda vez que la dirección del presente hacia el futuro es la dirección de la entropía, la orientación que señala la "flecha del tiempo" y que confiere a los procesos su carácter bistórico.

15 Guitton Jean, op.cit., p. 88.

${ }^{16}$ Idem, p. 80. 


\section{La concepción prigoginiana del tiempo}

En términos comparativos, la concepción prigoginiana de la temporalidad está más cerca de la idea agustiniana de historicidad que de la idea newtoniana de reversibilidad. El punto de semejanza entre Agustín y Prigogine se basa en la noción de extensio animi, no en la de intentio animi. Dicho de otro modo, la dirección irreversible del tiempo hacia el futuro -la extensio-, su papel constructivo, el carácter bistórico de todos los procesos, son los puntos de contacto entre ambas nociones de temporalidad. La extensio animi se vincula con la noción prigoginiana de irreversibilidad porque ambas señalan la dirección irrevrsible del presente bacia el futuro, la dirección de la entropía o "flecha del tiempo" que otorga a todos los procesos -naturales, humanossu carácter histórico.

Si la semejanza más notoria entre ambos autores se basa en esta noción, la diferencia más evidente radica en el concepto de intentio, idea vinculada a la trascendencia y a la eternidad que está ausente en Prigogine, ya que su concepción científica del tiempo es inmanentista y naturalista.

Para la física pre-prigoginiana (dinámica newtoniana, física cuántica y relativista), la "flecha del tiempo" que señala la dirección de la entropía y de la irreversibilidad fue considerada una ilusión, una apariencia producto de las limitaciones de nuestros sentidos, y fue reducida a ignorancia humana o aproximación. La dirección temporal que tiende al futuro es aquí irreal. La irreversibilidad es apariencia subjetiva, no tiene existencia física. En una conversación mantenida con Guitton, el científico León Brunschvicg le responde:

"Ha subrayado usted en su tesis un pensamiento de San Agustín según el cual habría dos ejes en el tiempo: uno que se dirige hacia el futuro, otro que se eleva hacia la eternidad. Este problema fue tratado posteriormente por Spinoza. Pero la idea de Spinoza (que yo comparto) es que la dirección que va hacia el futuro es ilusoria, imaginaria, infantil, provisoria, mítico"17.

La convicción de Brunschvicg es la creencia de la mayoría de los hombres de ciencia. Para el propio Einstein, inclusive, el tiempo irreversible es una ilusión producida por las limitaciones de nuestro cálculo y las

${ }^{17}$ Idem, pp. 88-89. 
restricciones de nuestros sentidos. Esta convicción es tan antiagustiniana como antiprigoginiana, en el sentido restringido de que ella contradice la existencia objetiva de la extensio y de la flecha del tiempo. La tensión hacia el futuro, la dirección temporal que señala el devenir, sería solamente un producto aparente, "imaginario", de nuestra subjetividad. No hay, para la física preprigoginiana, irreversibilidad real.

En la mayoría de sus textos, Prigogine reitera que el redescubrimiento de la irreversibilidad, que había sido sistemáticamente negada por la física pero afirmada por la filosofía, cierra la brecha abierta entre ambas disciplinas: el tiempo de la vida, el tiempo interior de la existencia humana analizado por los filósofos, es el mismo tiempo que el estudiado por la nueva física: el tiempo de todos los procesos irreversibles, de todos los sucesos o acontecimientos bistóricos. No se trata de "dos tiempos distintos" que no guardan entre sí relación alguna. Ni de dos dimensiones inconmensurables de la temporalidad. Por así decirlo, la misma flecha del tiempo que atraviesa la materia, nos atraviesa: todo envejece en la misma dirección. La entropía que determina la vejez y muerte de las estrellas, determina también nuestra propia vejez y nuestra propia muerte.

Para Prigogine, incluso el universo tiene una bistoria. A diferencia de Stephen Hawking, que concibe la creación y evolución del cosmos a partir del Big Bang como una construcción geométrica (aunque con un principio y un final en el tiempo ${ }^{18}$ ), Prigogine las concibe como un proceso esencialmente histórico, es decir, contingente e irreversible, producto de inestabilidades que generaron, a partir del caos, nuevos órdenes de mayor complejidad.

Pero, ¿en qué consiste exactamente esta concepción? ¿Cómo redefine el tiempo Prigogine? Para responder a esta cuestión, caracterizaré lo más detalladamente posible la secuencia conceptual que lo conduce a esta reconceptualización en termodinámica del no equilibrio y teoría del caos. La sucesión está vinculada a las nociones de inestabilidad, probabilidad y complejidad. Se asocia también con las ideas de entropia e irreversibilidad.

En primer término, Prigogine introduce la noción de "caos" en la

18 Hawking Stephen, Historia del tiempo. Del Big Bang a los agujeros negros, Crítica, México, 1988, Capítulo 2. 
ciencia, lo que implica la reformulación (ampliación) de las leyes de la naturaleza, no ya en términos extrahistóricos y universales, sino temporales $y$ locales. La inclusión del caos lo obliga a hablar de "leyes probabilistas" y a abandonar el determinismo y la reversibilidad resultantes de una legalidad que fuerza a la naturaleza a repetir ciegamente patrones de comportamiento, a iterar sus procesos. Esta concepción estrecha y reduccionista de "ley de la naturaleza", característica de la ciencia moderna, estaba ausente en Aristóteles:

"La idea de leyes de la naturaleza tiene una connotación legalista: parecería que la naturaleza está 'obligada' a seguir ciertas leyes. Destaquemos que en otras cosmovisiones dicha idea está ausente. Según Aristóteles, los seres vivos no están sometidos a leyes; su actividad es el resultado de causas internas autónomas, cada ser vivo se empeña en alcanzar su propia verdad inherente" 1 .

¿Cómo afecta esta idea de legalidad de la ciencia moderna a la noción de temporalidad? Claramente, suprimiendo la existencia objetiva de la flecha del tiempo, eliminando la dirección del devenir en las leyes fundamentales de la naturaleza, cancelando el papel constructivo del tiempo (en un mundo repetitivo y previsible, "tautológico", no hay novedad). Esto crea un abismo entre la ciencia clásica, cuyas leyes describen un mundo atemporal, y la experiencia humana más íntima según la cual percibimos (vivenciamos) que el pasado y el futuro no juegan el mismo papel en nuestra existencia, que la vida es esencialmente histórica e irreversible. Este abismo explica el cisma moderno entre ciencia y filosofía, y asimismo, el hiato entre ciencias naturales y humanidades.

El esquema conceptual de Prigogine es entonces el siguiente: Inestabilidad $\rightarrow$ Descripción Estadística $\rightarrow$ Irreversibilidad.

Este esquema implica que la ciencia debe incluir no sólo leyes, regularidades, sino también eventos, sucesos. La explicación científica narra de este modo una historia, no describe una geometria: la sucesión temporal irreversible de acontecimientos en los que el azar y las condiciones del entorno generaron

19 Prigogine Ilya, “¿El fin de la ciencia?”, en Nuevos paradigmas, cultura y subjetividad (compilado por Dora Fried Schnitman y traducido por Leonardo Walfson), Paidós, Buenos Aires, 1994, p. 38. 
un hecho contingente pero determinante, una vez acaecido. Azar y necesidad no se oponen: de ahora en más, son conceptos complementarios. Por eso Prigogine habla de "leyes del caos" y de "caos determinista".

Según él, la ciencia clásica buscaba certezas, y las certezas sólo se encuentran en un mundo estable, gobernado por leyes deterministas y reversibles, donde las nociones de azar, probabilidad, eventos estocásticos, están excluidas de la idea de "ley de la naturaleza". La negación del tiempo está vinculada a la búsqueda de certeza. La búsqueda de certeza, al determinismo. Este esquema conceptual sólo es realizable mediante la selección de sistemas aislados en equilibrio, o en sus proximidades. Estos sistemas se denominan "integrables", y constituyen los modelos del comportamiento dinámico de la física clásica.

Lejos del equilibrio, en cambio, la situación es muy distinta. Se producen "bifurcaciones", puntos de encrucijada donde la materia "elige" un rumbo u otro. Esta elección está sujeta al azar y a las probabilidades, e introduce un elemento histórico, contingente, en la descripción de los fenómenos físicos: sólo algunas posibilidades se actualizarán, no todas. Los puntos de bifurcación son zonas altamente inestables, muy sensibles a cualquier mínima alteración o modificación de su entorno. Las perturbaciones se amplifican una vez acaecidas. Esto se denomina "sensibilidad a las condiciones iniciales", y es la característica distintiva del caos, el sello de la inestabilidad más radical. Se vincula al concepto técnico de "resonancia" introducido por Poincaré para caracterizar los "sistemas no integrables". Bifurcación es, entonces, sinónimo de aleatoriedad, historicidad e irreversibilidad:

"Las bifurcaciones introducen en la descripción un elemento histórico. La elección entre las ramificaciones corresponde a un proceso aleatorio (...) Los puntos de bifurcación incluyen procesos estocásticos; son muy sensibles a las pequeñas perturbaciones" 20 .

Resumiendo, la ruptura de simetría temporal debida a la existencia de bifurcaciones posee un papel constructivo en los fenómenos de la naturaleza, que es esencialmente inestable. El tiempo no es una ilusión sino una construcción, una creación de novedad, como genialmente intuyó Bergson. En la misma sintonía, argumenta Prigogine:

"Creo justificado plantear que el tiempo es 'real' y que la irreversibilidad

${ }^{20}$ Idem, p. 50 
cumple en la naturaleza un papel constructivo fundamental. Por consiguiente, en nuestra descripción básica de la naturaleza es necesario que expongamos las raíces de la irreversibilidad. Ya no podemos seguir aceptando que el tiempo es una ilusión o el resultado de nuestro enfoque 'de grano grueso"'21.

\section{El encuentro entre San Agustín y Prigogine: acontecimiento, historicidad e irreversibilidad.}

La posición de Prigogine se emparenta con la crítica filosófica a la concepción estática de la temporalidad sostenida por la ciencia moderna. Bergson, por ejemplo, ha cuestionado duramente esa temporalidad sin tiempo, ese tiempo reducido a parámetro geométrico del movimiento, tiempo externo y reversible que poco y nada tiene que ver con la vivencia existencial de la temporalidad, dramáticamente descrita por San Agustín. Ese tiempo humano, interior e irreversible, está más cerca del tiempo -complejidad de Prigogine, que es también intrínsecamente bistórico y está sujeto a fluctuaciones que generan incertidumbre. Esto no significa, obviamente, que la extensio animi, por la cual el yo tiende a lo que ain no es, la dirección o el sentido del deseo, se asemeje totalmente a los procesos físicos irreversibles en los que el azar y las condiciones de contorno generan un dinamismo y una "deriva", un comportamiento direccionado globalmenmte coherente. No significa, tampoco, que una concepción inmanentista y naturalista se asemeje a una postura espiritualista e interiorista. Pero sí significa que ambas concepciones de la temporalidad concuerdan en las nociones de irreversibilidad, acontecimiento e bistoricidad, pese a sus innumerables diferencias.

La concepción newtoniana del tiempo, por el contrario, supone una posición diferente: "en cada instante todo viene dado" significa sin más que el establecimiento de las condiciones iniciales de un sistema dinámico permite deducir, en cada instante, todo el futuro del sistema, y también, retrodecir todo su pasado, ya que ambos estados, futuro y pasado, son simétricos y juegan por tanto el mismo papel en las ecuaciones dinámicas. En la descripción física clásica no hay "flecha del tiempo". El movimiento es reversible. Sus ecuaciones son invariantes con respecto a la inversión del tiempo. Pasado y futuro son equivalentes, y por tanto, intercambiables en las ecuaciones

${ }^{21}$ Idem, p. 57 
dinámicas que describen el movimiento.

Prigogine afirma que esa especie de "instante eterno" que definió Newton, y que excluye al devenir en tanto dirección privilegiada del tiempo, implica una perspectiva opuesta a la de San Agustín. La dialéctica agustiniana entre el presente del pasado (la memoria), el presente del presente (la atención), y el presente del futuro (la expectativa), se revela totalmente opuesta a la concepción newtoniana del tiempo presente, puesto que rompe la simetría entre el pasado y el futuro, y afirma sin rodeos la irreversibilidad del tiempo humano; ruptura de simetría e irreversibilidad que la teoría del caos redescubre por doquier en la naturaleza. Sus leyes estadísticas ponen de manifiesto que el tiempo bumano y el tiempo de los procesos fisicoquímicos, el tiempo de los fenómenos naturales que evolucionan hacia el futuro donde la entropía aumenta continuamente, no son tiempos antagónicos e irreconciliables, ya que todos los procesos naturales -incluidos los procesos humanos- están atravesados por la misma y única "flecha del tiempo" que atraviesa el universo. Prigogine admite una multiplicidad de tiempos, de escalas de tiempo, pero a condición de sumergirlos en la corriente temporal única de la irreversibilidad, en el cauce universal del devenir, ese "río" que ni se detiene ni retrocede, y que es en cada instante distinto, nuevo, como afirmaba Heráclito.

El tiempo newtoniano, en cambio, es único: un parámetro geométrico y externo del movimiento. En contrapartida, el tiempo de San Agustín, como el de Prigogine, no es uniforme sino múltiple, diverso (heterogeneidad o complejidad que en San Agustín surge de la dialéctica entre la memoria, la atención y la expectativa, y en Prigogine, de la secuencia conceptual inestabilidad $\rightarrow$ ruptura de simetría $\rightarrow$ probabilidad $\rightarrow$ irreversibilidad). En ambos, el tiempo tiene un papel constructivo. En esta creatividad reside la imprevisibilidad o indeterminación del tiempo. Prigogine asumirá el carácter probabilista de las leyes de evolución temporal mediante la descripción estadística; San Agustín destacará el carácter dramático de la existencia humana debido a la irreversibilidad del tiempo y a la imposibilidad de prever el futuro, un futuro que nos depara sorpresas impensadas y que está muy lejos del futuro deducible, tautológico, casi tedioso, de Newton. Solamente en un mundo incierto e inestable la vida adquiere un carácter dramático. En este mundo, el azar, el acontecimiento, la irreversibilidad y la historicidad, son conceptos inherentes a la noción de temporalidad. Y esta noción afecta tanto a los seres humanos como a los procesos de la naturaleza, según la teoría del caos. 
Prigogine procura terminar con el divorcio epistemológico entre la ciencia y la filosofía proponiendo un modelo coherente para pensar el tiempo sin escindir al bombre de la naturaleza de la que forma parte, sin contraponer el tiempo bumano al tiempo físico como si se tratara de dos dimensiones antagónicas e inconmensurables de la temporalidad. El final de este divorcio sólo es posible si la nueva ciencia asume cabalmente el indeterminismo, que es el efecto de la inestabilidad y el caos, y si acepta sin rodeos el papel constructivo del tiempo en sus leyes, la existencia objetiva de la flecha del tiempo común a todo el universo:

“Hoy no tenemos temor de la 'hipótesis indeterminista'. Es la consecuencia natural de la teoría moderna de la inestabilidad y el caos. Y otorga una significación física fundamental a la flecha del tiempo, sin la cual somos incapaces de entender los dos rasgos principales de la naturaleza: su unidad y su diversidad. La flecha del tiempo, común a todas las partes del universo, da testimonio de dicha unidad. Tu futuro es mi futuro, el futuro del sol y de cualquier otra estrella (...) Ninguna formulación de las leyes de la física que ignore el papel constructivo del tiempo podrá jamás satisfacer nuestra necesidad de entender la naturaleza" 22 .

Como el tiempo y la realidad están íntimamente ligados, negar el tiempo significa negar la realidad, aunque en esta negación haya residido el triunfo de la física clásica, su impresionante éxito durante tres siglos. Este éxito se basó esencialmente en la supresión de la irreversibilidad y en la simplificación de la complejidad (esto es, en la negación de la diversidad). Reenconrtar en la naturaleza las raíces del tiempo es el objetivo declarado de la teoría de Prigogine. Pero esto no significa, sin más, que el autor pretenda resolver definitivamente el misterio del tiempo, misterio que oscila entre lo que está en el tiempo y lo que está fuera del tiempo, entre el tiempo y la eternidad:

"Los problemas de los límites del determinismo, del azar y de la irreversibilidad y la noción de realidad están estrechamente vinculados, y comenzamos a ver cómo se relacionan. Conforme somos capaces de hallar en la naturaleza las raíces del tiempo, éste deja de ser el concepto que separa al hombre de la misma, Ahora expresa nuestra pertenencia a la naturaleza, no nuestra alienación. La visión del mundo que nos rodea converge con la del mundo interior (...) Probablemente continúe para siempre la dialéctica entre

${ }^{22}$ Prigogine Ilya, El fin de las certidumbres. (trad. de Pierre Jacomet), Andrés Bello, Santiago de Chile, 1997, p. 61. 
lo que está en el tiempo y lo que está fuera del tiempo. Pero quizá estemos ahora en un momento favorable en el que comenzamos a percibir mejor la convergencia, la transición entre reposo y movimiento, entre el tiempo detenido y el tiempo en decurso" 23 .

La visión del mundo que nos rodea converge con la del mundo interior: esta expresión crucial implica que la visión del tiempo físico, del tiempo exterior y objetivo, converge con la visión del tiempo interior, del tiempo bumano tal como es vivenciado. Duración, expectativa, improvisación, creación, novedad, acontecimiento, irreversibilidad e historia (impronta o huella del pasado en el presente, memoria del ayer en el hoy ) son algunas de las categorías que nos permiten repensar el tiempo, ese tiempo múltiple de diversas escalas internas pero cuya direccionalidad es única, universal: la orientación del devenir señalada por la extensio animi agustiniana y por la flecha del tiempo en la teoría del caos.

Pero forjar una nueva noción de tiempo capaz de transcender las categorías del devenir y de la eternidad; recategorizar los conceptos necesarios para resolver el misterio del tiempo conciliando sus aspectos de multiplicidad y unidad, es más bien un proyecto o una expresión de deseos que una obra consumada. Ni San Agustín ni Prigogine han resuelto definitivamente el problema del tiempo, pero ambos han iluminado, cada uno a su manera y desde sus perspectivas - diferentes pero complementarias- la oscuridad de este misterio.

\section{Resumen}

El trabajo compara las nociones de "historicidad" e "irreversibilidad" en la concepción del tiempo de Prigogine y de San Agustín, estableciendo semejanzas y diferencias. Analiza primero la idea agustiniana de la temporalidad siguiendo el Libro Undécimo de las Confesiones, y las interpretaciones de dicha idea efectuadas por Paul Ricoeur y por Jean Guitton. A continuación, analiza la redefinición del tiempo llevada a cabo por Ilya Prigogine, diferenciándola fundamentalmente de la concepción estática o geométrica de la dinámica newtoniana. Muestra que esta reconceptualización lo diferencia de la física clásica y lo acerca a la idea agustiniana del tiempo, en tanto y en cuanto, en ambos autores, las nociones de "irreversibilidad", "historicidad", "novedad", "acontecimiento" y "creatividad", son

23 Prigogine Ilya, ¿Tan sólo ina ilusión? Una exploración del caos al orden (trad. de Francisco Martín), Tusquets, Barcelona, 1997, pp. 38-39. 
cruciales para entender la temporalidad. Finalmente, pone de manifiesto las diferencias entre los dos autores: mientras que San Agustín se refiere fundamentalmente a un tiempo humano o existencial, en el que está presente un claro sentido de la trascendencia, Prigogine alude al tiempo de la dinámica y la termodinámica de los procesos irreversibles alejados del equilibrio, en un sentido ciertamente inmanente y físico.

Palabras claves: "tiempo", "irreversibilidad", "San Agustín”, "I. Prigogine”.

\begin{abstract}
The essay compares the notions of "historicity" and "irreversibility" in the Prigoginean and Agustinian conceptions, establishing likenesses and differences. It analyzes the Agustinian idea of the temporality following the Book Eleventh of the Confessions, and the interpretations of Paul Ricocur and Jean Guitton. It analyzes, next, the redefinition of the "time" formulated by Ilya Prigogine, differentiating it, fundamentally, from the static or geometric conception of the Newton's dynamic. It shows that this re-conceptualization differentiates it from the classic Physics and brings it nearer to the Agustinian idea of the time, as that, in both authors, the "irreversibility", "historicity", "novelty", "event" and "creativity" notions are crucial to understand the temporality. Finally, it is shown here the differences between both authors: while St. Agustin fundamentally refers to a human or existential time, in which a clearing sense of the transcendency is present, Prigogine refers to the time of the dynamics and the thermodynamics, of the irreversible processes receded from the equilibrium, and certainly in a physical immanent sense.
\end{abstract}

Key words: "time”, "irreversibility", "St. Augustin", "I. Prigogine”. 